\title{
A review of astrophysics experiments on intense lasers*
}

\author{
Bruce A. Remington ${ }^{\dagger, a)}$ \\ Lawrence Livermore National Laboratory, Livermore, California 94550 \\ R. Paul Drake ${ }^{\text {b) }}$ \\ University of Michigan, Ann Arbor, Michigan 48109 \\ Hideaki Takabe ${ }^{\text {c) }}$ \\ Osaka University, Osaka, Japan \\ David Arnett ${ }^{\mathrm{d})}$ \\ University of Arizona, Tucson, Arizona 85721
}

(Received 18 November 1999; accepted 31 January 2000)

\begin{abstract}
Astrophysics has traditionally been pursued at astronomical observatories and on theorists' computers. Observations record images from space, and theoretical models are developed to explain the observations. A component often missing has been the ability to test theories and models in an experimental setting where the initial and final states are well characterized. Intense lasers are now being used to recreate aspects of astrophysical phenomena in the laboratory, allowing the creation of experimental testbeds where theory and modeling can be quantitatively tested against data. We describe here several areas of astrophysics-supernovae, supernova remnants, gamma-ray bursts, and giant planets-where laser experiments are under development to test our understanding of these phenomena. (C) 2000 American Institute of Physics. [S1070-664X(00)97605-5]
\end{abstract}

\section{INTRODUCTION}

Modern intense lasers produce energy densities in submillimeter-scale volumes large enough to access phenomena that otherwise appear only in energetic astrophysical systems. Matter can be reproducibly prepared in conditions that are equivalent, in a rigorously scaled sense, to those in large astrophysical systems. Examples of areas that can be studied include: strong shock phenomena, high Mach number jets, strongly coupled plasmas, compressible hydrodynamic instabilities, radiation flow, photoevaporation front hydrodynamics, and fundamental properties such as opacities and equations of state. Consequently, a vibrant new field of research is emerging-laboratory astrophysics with intense lasers. ${ }^{1}$

Traditional laboratory astrophysics has generally focused on measuring fundamental "input" parameters such as nuclear reaction cross sections and opacities. These fundamental "input" quantities are required in models of phenomena such as stellar pulsations and supernova light curves. A new category of laser astrophysics experiments is aimed at probing astrophysical dynamics directly by creating scaled reproductions of the astrophysical systems in the laboratory. This allows the "output" of astrophysics theories and modeling to be tested directly, where the initial and final states are well characterized. We present a brief review of the emerging field of "laser astrophysics," selecting experiments for discussion that are relevant to: supernovae (Sec.

\footnotetext{
*Paper AR1 A Bull. Am. Phys. Soc. 44, 17 (1999).

'Review speaker.

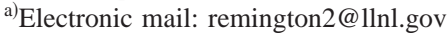

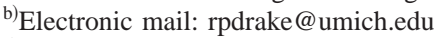

${ }^{c}$ Electronic mail: takabe@ile.osaka-u.ac.jp

d)Electronic mail: dave@bohr.as.arizona.edu
}

II), supernova remnants (Sec. III), gamma ray bursts (Sec. IV), and the giant planets. (Sec. V).

\section{SUPERNOVAE}

Core-collapse supernovae represent the dramatic endpoint of one of nature's most impressive cycles: the life and death of a massive star. ${ }^{2-6}$ The final death throes of the star are spent in a high-stakes "tug of war" pitting quantum mechanical degeneracy pressure against the more familiar gravitational pressure. The outcome determines whether the final state of the star is a white dwarf, neutron star, or black hole, and is based on the strength of the degeneracy pressure to withstand the radially inward tug of gravity. ${ }^{7}$ Stars with initial masses of $1-8 M_{\odot}$ (where $M_{\odot}$ corresponds to the mass of the sun) finish their hydrogen burning while their cores are not yet degenerate. They undergo core contraction, which raises the core density and temperature sufficiently to trigger He burning. These stars subsequently loose mass effectively, and end their lifetimes as white dwarfs, with masses of $\sim 0.6 M_{\odot}$. White dwarfs are supported by the pressure of the degenerate electrons in their interiors, that is, it is the quantum mechanical Pauli exclusion principle that prevents further collapse. The maximum mass possible for a white dwarf is the Chandrashekar limiting mass, $M_{\mathrm{Ch}}$ $\approx 1.4 M_{\odot}$. Larger stars have high enough temperatures in their cores to continue the nuclear fusion burning cycle up to $\mathrm{Fe}$. Once the core reaches $\mathrm{Fe}$, the nuclear fusion reactions no longer release net energy (because the nuclear binding energy per nucleon is maximum in $\mathrm{Fe}$, at nearly $9 \mathrm{MeV}$ / nucleon), and the thermonuclear fires are extinguished. The mass of the Fe core continues to grow as the surrounding layers burn their way to this thermonuclear endpoint until the 
Fe core mass exceeds $\sim 1.4 M_{\odot}$. At this point, there is no longer sufficient heat produced in the core to balance cooling by neutrino emission and photonuclear dissociation, and the core surrenders to gravity, triggering a catastrophic gravitational collapse that is over in a matter of seconds. This collapse is arrested only when the core density reaches that of degenerate nuclear matter $\left(\sim 2 \times 10^{14} \mathrm{~g} / \mathrm{cm}^{3}\right)$. The Fermi degeneracy pressure, $P_{\mathrm{deg}} \sim \rho^{2 / 3}$, increases sufficiently to stop the implosion, and a spectacular nuclear rebound occurs whose strength is determined by the equation of state (EOS) of bulk nuclear matter. By a mechanism still debated, this launches the powerful outward-propagating shock that first "stalls" in the infalling matter, then gets re-energized by convection and by energy deposition due to neutrinos emitted from the core. Collective plasma effects may be involved in the neutrino energy deposition. ${ }^{8}$ The shock thus restarted, traverses the overlaying layers and effectively blows the star apart. Thus, the catastrophic end of the stellar core marks the spectacular beginning of a core-collapse supernova. This explosive birth is observed as a bright flash of UV light, followed by an extended period of enormous luminosity, as illustrated by the light curve for SN1987A in Fig. 1(a). ${ }^{4,9,10}$ If the core has a mass larger than $2-3 M_{\odot}$, the core collapse continues to form a black hole, otherwise a neutron star is formed.

\section{A. Supernova light curves}

The visual supernova $(\mathrm{SN})$ commences when the shock breaks out through the surface of the star about an hour after the core collapses, ${ }^{4}$ as shown in the inset of Fig. 1(a) from a calculation for SN1987A. There is a sudden increase in effective temperature to $20-40 \mathrm{eV}$ (Ref. 10) and luminosity, followed by a rapid drop in both quantities, as the star expands and cools adiabatically. [The effective temperature (or "brightness temperature") $T_{\text {eff }}$ is defined by $L$ $=4 \pi R_{\text {photosphere }}^{2} \sigma T_{\text {eff }}^{4}$; where $L$ is the luminosity ( $\left.\mathrm{erg} / \mathrm{s}\right)$, $R_{\text {photosphere }}$ is the radius of the photosphere, and $\sigma$ is the Stefan-Boltzmann constant. The color temperature is defined by the spectral shape, and $T_{\text {color }}=(2-3) T_{\text {eff }}$ for these conditions. ${ }^{11}$ ] About 30 min after shock breakout, the luminosity approaches a constant value, as the recombination front, which determines the photosphere, moves inward in mass at roughly the constant temperature (for hydrogen) of $6000 \mathrm{~K}$. After some 20-40 days, the heat from the radioactive core, heated by Compton scattering of the $\gamma$-rays produced from ${ }^{56} \mathrm{Ni},{ }^{56} \mathrm{Co}$, and ${ }^{44} \mathrm{Ti}$, reaches the photosphere, and the light curve rises up in a broad secondary maximum [Fig. 1(a)]. Subsequently, the decay of the light curve is monotonic in time at a rate determined by the half-lives of the various radioactive nuclei that serve as the heat source. As the hot core nears the surface of the star, $\mathrm{x}$-rays and $\gamma$-rays were observed directly by satellite observations (Ginga/Mir) and various balloon-born experiments (SMM, LM, CIT, FG). The light curve contains a wealth of information about the star and its explosion. The luminosity varies directly with the explosion energy per unit mass, $E / M$, and also depends on the initial radius of the star. For the same $E / M, \mathrm{SN}$ from small stars are not as bright, since more energy goes into hydrodynamic expansion. The luminosity is (a)

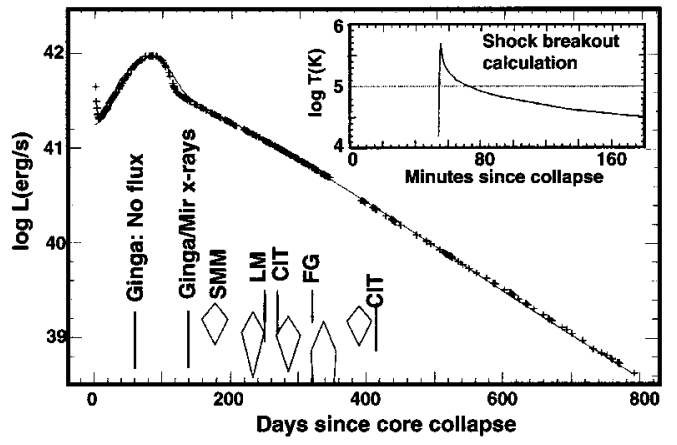

(b)

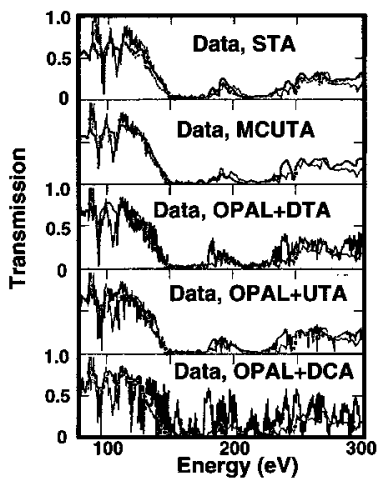

(c)
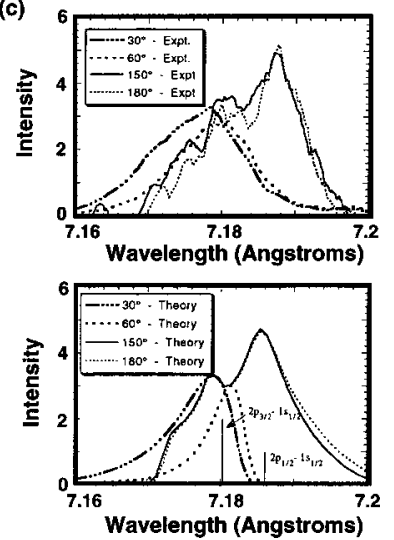

FIG. 1. Supernova light curves and opacities. (a) Light curve for SN1987A (reproduced from Ref. 4). The '+' symbols are the observed light curve, and the thin solid line is an analytic model described in Ref. 4. The different dates indicated show when x-rays were first detected on day 139 by the Ginga/Mir experiment, when $\gamma$-rays from ${ }^{56} \mathrm{Co}$ were detected by the Solar Maximum Mission (SMM) at day 178, and when subsequent detections of $\gamma$-rays occurred by several balloon experiments (CIT, LM, FG). The inset shows a calculation of the evolution of temperature versus time as the shock breaks out the surface of the star. (b) Modeling and experimental measurements of the opacity of $\mathrm{Fe}$ at a temperature of $T=59 \mathrm{eV}$ and $\rho$ $=11 \mathrm{mg} / \mathrm{cm}^{3}$ (reproduced from Ref. 16). (c) Experimental measurements of radiation line transport through an expanding $\mathrm{Al}$ plasma with a large velocity gradient (reproduced from Ref. 24).

on average inversely proportional to the opacity, since lower opacity means shorter radiative diffusion times. Finally, the light curve time evolution is sensitive to the degree that the core hydrodynamically mixes outwards into the envelope, bringing heat nearer to the photosphere. An ability to quantitatively calculate an SN light curve would allow the intrinsic brightness of the $\mathrm{SN}$ to be known. Comparison with the observed brightness would give its distance through the expanding photosphere method. ${ }^{12,13}$ Together with spectroscopic measurements of its red shift, this allows the Hubble constant, $H_{0}$, to be determined. ${ }^{14}$ There are several aspects to synthetic light curve calculations that could benefit from laboratory experiments, such as radiation flow, opacities, and hydrodynamic mixing.

Exploding stars create a homologous expansion, where each radiating region resides in a velocity gradient and sees plasma receding from it in all directions. In other words, the absorbing regions are always red shifted relative to the emitting regions. For photons emitted in one region to escape the star, they have to pass through "windows" in opacity, where the absorption probability is low. To be able to construct a synthetic light curve requires (1) access to high quality 
("static") opacity tables, and (2) a radiation transport calculation including the effects of the Doppler shifts in the opacity line and edge locations, due to the expansion. The opacity tables are produced by calculations with sophisticated opacity codes such as OPAL. ${ }^{15}$

Experiments have been conducted on various lasers ${ }^{16-22}$ to measure the local thermonuclear equilibrium (LTE) opacities of a variety of materials (e.g., Fe, Ge, Na, Al) at temperatures in the range of $10-75 \mathrm{eV}$ and densities of 10-50 $\mathrm{mg} / \mathrm{cm}^{3}$. As an example, we show in Fig. 1(b) the results of a measurement of the opacity of $\mathrm{Fe}$ at $T_{c}-59 \mathrm{eV}$ and $\rho$ $=11 \mathrm{mg} / \mathrm{cm}^{3}$, where the Fe sample was radiatively heated in a laser-driven hohlraum. ${ }^{16}$ The measurement was made using an imaging, time-resolved, grating spectrometer. The thin, tamped Fe foil contained an admixture of $\mathrm{Na}$, whose opacity was measured simultaneously with the Fe opacity. The 1-D radiographic spatial imaging gives the sample density. The spectrum of the low-Z Na dopant, when compared with OPAL calculations, gives the electron temperature. Hence, the opacity of Fe was measured for known conditions of $T_{c}$ and $\rho$. (The experimental results shown in Fig. 1(b) are compared with several different opacity calculations employing different approximations.) The conclusion of this work was an unambiguous demonstration of the need to include term splitting in the opacity calculations. Models that neglect this, such as DCA, significantly underpredict the opacity. Complementary experiments are also being developed on a Z-pinch facility to test LTE opacity codes at lower densities, where there are greater differences between codes. ${ }^{23}$

Another experiment measured radiation line transport in an expanding plasma ${ }^{24}$ [Fig. 1(c)]. The experiment studied the structure of a doublet in the aluminum spectrum, at a wavelength near 7.18 $\AA$. The emission occurs from an optically thick plasma with a significant velocity gradient, so that emission in one line is often absorbed and re-emitted by the other line at another location in the plasma. The resulting line structure is complex, but can be reproduced by modeling only when this expansion effect on the radiation transport is taken into account. Hence, experiments are under development to test opacity calculations, both static and in expansion, aspects of which are relevant to SN light curves.

The 1-D modeling of light curves such as those for SN1987A, even with the most sophisticated opacities, still fail to reproduce the time evolution. It appears that additional dynamics is at work. The modeling used to successfully reproduce the light curve for SN1987A shown in Fig. 1(a) assumes that the radioactive $\mathrm{Ni}$, while centrally concentrated, was distributed half-way to the surface of the star. ${ }^{4}$ This suggests that large scale hydrodynamic mixing had to have occurred after the ${ }^{56} \mathrm{Ni}$ was synthesized in the core in the explosion. Hence, hydrodynamic instabilities appear to be an important ingredient in the dynamics of SN.

\section{B. Instabilities in the explosion phase}

A core-collapse $\mathrm{SN}$ is driven by an extremely powerful shock, and strong shocks are the breeding ground of hydrodynamic instabilities, such as the Rayleigh-Taylor (RT) and Richtmyer-Meshkov (RM) instabilities. The Rayleigh-
Taylor instability occurs when a lower-density fluid accelerates a higher-density fluid. The Richtmyer-Meshkov instability is closely related, with the role of gravity replaced by the inertia from an impulsive acceleration due to a shock wave.

During the shock transit phase, the RM instability is triggered at each discontinuity in the density profile of the star, i.e., at the $\mathrm{O}-\mathrm{He}$ and $\mathrm{He}-\mathrm{H}$ ' interfaces.' After shock transit, hydrodynamic mixing continues due to the RT instability, as the denser layers are decelerated by the lower-density outer layers. The outward mixing of the higher-density, radioactive core material (e.g., ${ }^{56} \mathrm{Ni},{ }^{56} \mathrm{Co},{ }^{44} \mathrm{Ti}$ ) brings the radioactive heat source toward the surface of the star. These explosion products decay by the emission of $\gamma$-rays, which Compton scatter off electrons in their vicinity. This reheats the photosphere and causes the secondary maximum in the light curve at 40-120 days [Fig. 1(a)]. The RT mixing induces this reinvigoration of the light curve to start earlier, broadening the secondary maximum. Observations of the light curve of SN1987A unambiguously showed this broadening of the secondary peak, suggesting enhanced transport from the core out to the photosphere. ${ }^{2,3}$ Two-dimensional calculations of the development of the mixing at the $\mathrm{O}-\mathrm{He}$ and $\mathrm{He}-\mathrm{H}$ interfaces using the supernova code PROMETHEUS $^{25,26}$ show that spikes of denser oxygen, and helium penetrate outward into the less dense envelope of hydrogen, while bubbles of hydrogen move inward relative to the average location of the H/He boundary [Fig. 2(a)]. This interpenetration occurs through the growth and nonlinear evolution of the RT instability.

Laser-based experiments can generate strong shockinitiated nonlinear hydrodynamic mixing conditions similar to those found in SNe. In a set of experiments scaled to reproduce the hydrodynamics of the $\mathrm{He}-\mathrm{H}$ interface of SN1987A about an hour after explosion, a strong shock was passed through an interface separating dense "core" material $(\mathrm{Cu})$ from the lower density outer envelope $\left(\mathrm{CH}_{2}\right) \cdot{ }^{27,28} \mathrm{~A}$ 2-D sinusoidal ripple (1-D wave vector) was imposed at the interface. The subsequent 2-D growth due to the RM and RT instabilities was measured by $\mathrm{x}$-ray backlighting. Spikes of $\mathrm{Cu}$ penetrating upward into less-dense $\mathrm{CH}_{2}$ were observed as a consequence of the RT instability [Fig. 2(b)]. This interpenetration was calculated in $2 \mathrm{D}$ with PROMETHEUS and the simulations reproduced the observations very well.

A theoretical look at the relation between the hydrodynamics occurring in the $\mathrm{SN}$ versus in the laboratory experiment shows that a rigorous mapping exists. Consider the $\mathrm{He}-\mathrm{H}$ interface at $1600 \mathrm{~s}$ in the $\mathrm{SN}$ and the $\mathrm{Cu}-\mathrm{CH}$ interface at $20 \mathrm{~ns}$ in the laser experiment. In both settings, the Reynolds number (the ratio of the inertial to the viscous force) and the Peclet number (the ratio of the convective to the conductive heat transport) are large. Therefore, viscosity and thermal diffusivity are negligible, and the dynamics of the interface are well described by Euler's equations for a polytropic gas: ${ }^{29}$ 
(a)

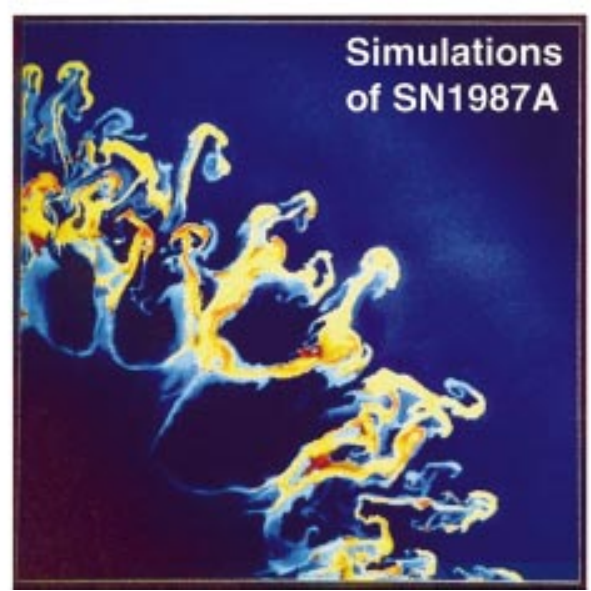

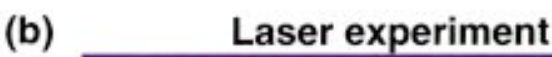

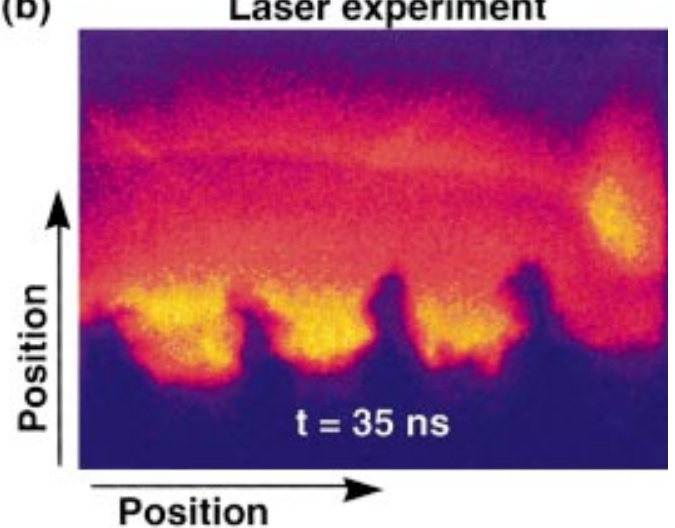

FIG. 2. (Color) Mixing in supernova explosion hydrodynamics. (a) Image of simulated hydrodynamic mixing from SN1987A at $t=12000 \mathrm{~s}$ (reproduced from Ref. 25). (b) An image from a laser experiment designed to measure this hydrodynamic mixing of a $\lambda=200 \mu \mathrm{m}$ wavelength ripple under scaled conditions at $t=35 \mathrm{~ns}$ (reproduced from Ref. 1).

$$
\begin{aligned}
& \rho\left(\frac{\partial \mathbf{v}}{\partial t}+\mathbf{v} \cdot \boldsymbol{\nabla} \mathbf{v}\right)=-\nabla p, \\
& \frac{\partial \rho}{\partial t}+\nabla \cdot(\rho \mathbf{v})=0,
\end{aligned}
$$

and

$$
\frac{\partial p}{\partial t}-\gamma \frac{p}{\rho} \frac{\partial \rho}{\partial t}+\mathbf{v} \cdot \nabla \rho-\gamma \frac{p}{\rho} \mathbf{v} \cdot \nabla \rho=0 .
$$

which represent conservation of momentum, mass, and entropy, respectively. It is straightforward to show by substitution that Eq. (1) is invariant under the following scale transformation:

$$
\begin{aligned}
& h_{\mathrm{SN}} \rightarrow a h_{\mathrm{lab}}, \\
& \rho_{\mathrm{SN}} \longrightarrow b \rho_{\mathrm{lab}}, \\
& p_{\mathrm{SN}} \longrightarrow c p_{\mathrm{lab}}, \\
& \tau_{\mathrm{SN}} \longrightarrow a(b / c)^{1 / 2} \tau_{\mathrm{lab}},
\end{aligned}
$$

where $h, \rho, p$, and $\tau$ correspond to characteristic spatial, density, pressure, and time scales, and subscripts $\mathrm{SN}$ and lab refer to the supernova and laboratory laser experiment, respectively. When transformation (2) is inserted into Eq. (1), the constants $a, b$, and $c$ cancel, and the dynamics described by Euler's equation are indistinguishable in the SN and the laser experiment. Both settings are probing the same physics. Any insights gained through the laser experiment apply directly to the $\mathrm{SN}$ through the mapping described by Eq. (2). For example, the hydrodynamics illustrated in Figs. 2(a) and 2(b) are similar, and can be related through the SN-tolaboratory mapping of $h, \rho, p, \tau$, and $g=\nabla p / \rho$ [Eq. (2)] giving $10^{11} \mathrm{~cm}$ to $50 \mu \mathrm{m}, 8 \times 10^{-3} \mathrm{~g} / \mathrm{cm}^{3}$ to $4 \mathrm{~g} / \mathrm{cm}^{3}, 40$ to $0.6 \mathrm{Mbar}$, and $10 g_{0}$ to $10^{10} g_{0}$, where these values were taken at times of $2000 \mathrm{~s}$ for the $\mathrm{SN}$ and $20 \mathrm{~ns}$ for the laboratory experiment. ${ }^{29}$ Here, $g_{0}$ refers to the acceleration due to gravity at the surface of the earth.

An example where laboratory experiments can generate valuable insights relative to the star is the comparison of RT instability growth in 2D versus 3D. PROMETHEUS simulations comparing the evolution of RT bubbles and spikes in two and three dimensions in a proposed laboratory experiment show that single-mode 3D perturbations should penetrate $30 \%-50 \%$ farther than those in 2D. Initial laboratory results confirm this difference for single-mode perturbations. ${ }^{30}$

\section{SUPERNOVA REMNANTS}

While SN explosions mark the end of a massive star, they also mark the beginning of its new life as a supernova remnant (SNR). Well-known examples of SNRs such as the remnants of Tycho's $\mathrm{SN}^{31}{ }^{31}$ Keplers $\mathrm{SN},{ }^{32}$ the Cygnus loop, ${ }^{33}$ $\mathrm{SN}_{1006}{ }^{34}$ and the Crab nebula ${ }^{35}$ provide exquisite visual testimony to their violent births. There are several active areas of research regarding the dynamics and evolution of SNRs which may be better understood with laser experiments.

Shock dynamics dominate the evolution of supernova remnants (SNRs). The rapidly expanding ejecta from the supernova drive a shock forward into the surrounding medium, and a reverse shock forms where the ejecta are decelerated by the accumulating, shocked matter. The place where the ejecta and ambient medium meet, called the contact discontinuity, becomes hydrodynamically unstable. Currently the most actively observed SNR is the young remnant forming around SN1987A. This remnant consists of the standard SN ejecta expanding into the ambient medium, as well as a mysterious inner and two outer circumstellar nebular rings, which apparently existed prior to the SN explosion. Various models have been proposed for these rings, but as of yet no explanation fully explains their origin. The SN ejecta, however, are moving very fast $\left(\sim 10^{4} \mathrm{~km} / \mathrm{s}\right)$ compared to the nearly static $(\sim 10 \mathrm{~km} / \mathrm{s})$ inner ring, which has a diameter of $\sim 1$ light year. It is widely expected that the ejecta-forwardshock system will impact the inner edge of the inner ring within the next $\sim 5$ years. This should launch a strong shock into the ring, heating it to $100-300 \mathrm{eV}$ temperatures, and cause emissions at all wavelengths, from optical to x-ray. ${ }^{36,37}$ Careful observation of this impact should shed light on the 
(a)

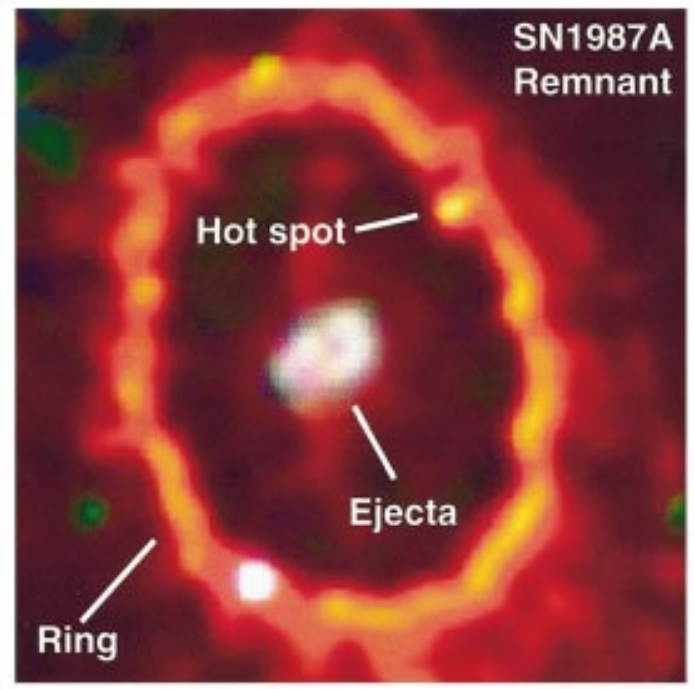

(b)

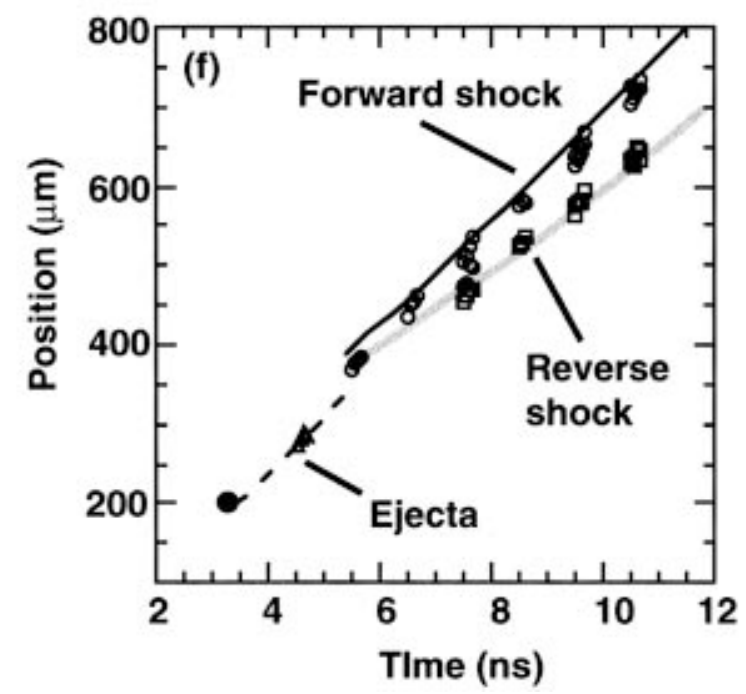

FIG. 3. (Color) Young supernova remnant dynamics. (a) Observational image of the inner circumstellar ring of SN1987A (Ref. 38 and http:// antwrp.gsfc.nasa.gov/apod/ap980217.html). (b) Image from shock experiments designed to produce similar, scaled regimes of strong shock hydrodynamics (reproduced from Ref. 40).

structure, composition, and hopefully origin of the rings. Recent images of the inner ring ${ }^{38}$ show a rapidly brightening, localized hot spot [upper right corner of Fig. 3(a)], suggesting that the collision of the forward shock with the ring has actually started. Spectral imaging of Lyman- $\alpha$ radiation, which is produced at the reverse shock, indicate that the reverse shock has traversed about $70 \%$ of the distance from the ring to the star. ${ }^{38}$

Laser experiments can produce shock structures similar to those in an SNR, under well-scaled hydrodynamic conditions. ${ }^{29,39-41}$ Experiments have been developed in 1D to reproduce the basic dynamics of SNR formation: fastmoving shock-induced ejecta sweeping into a surrounding low density, static ambient atmosphere. This launches a forward shock into the ambient medium and a reverse shock into the stagnating ejecta [Fig. 3(b)], much like the dynamics of SNR formation. Indeed, the laboratory experiment can be modeled by the self-similar model of Chevalier ${ }^{42}$ developed to describe the 1D dynamics of SNRs.

Two-dimensional experiments have commenced to examine the hydrodynamic instabilities at the contact discontinuity. One of the driving motivations for studying SNR physics relevant to SN1987A is the much anticipated impact of the SN blast wave with the inner circumstellar nebular ring. The interaction of the shock with the ring is sure to be rich in $3 \mathrm{D}$, strong shock effects. A laser experiment is being developed to elucidate the 3D nature of the interaction of a strong shock with a localized high density feature such as a sphere. $^{43}$ The 3D development strongly affects the interactions, with azimuthal (3D) modes growing, and enhancing the "shredding", of the sphere. A similar 3D effect is likely for the interaction of the SN1987A blast wave with the inner ring, and in shock-cloud interactions in general. ${ }^{44}$

Under the current conditions for the remnant of SN1987A, the scale transformation based on Euler's equa- tions described above for the explosion hydrodynamics might be applied again. For this to be relevant, one has to consider whether the shock is radiative, and whether the ambient magnetic field localizes the plasma. For the current conditions of SN1987A, the plasma density is low enough that the shocks are not radiative, that is, the radiative cooling time scale is long compared to a hydrodynamic time scale, $\tau_{\text {rad }} / \tau_{\text {hydro }} \gg 1$. Also, the ambient magnetic field, $B=\sim 100 \mu$ Gauss, is large enough that the ion Larmor radius is much smaller than spatial scales of interest. Hence, the plasma can be treated hydrodynamically, the dynamics can be treated again with Euler's equations, Eq. (1), and the same rigorous scale transformation [Eq. (2)] holds. For the SNR-to-laboratory transformation corresponding to the $1 \mathrm{D}$ experiment shown in Fig. 3(b), we get 0.03 light year mapping to $100 \mu \mathrm{m}, 10^{4} \mathrm{~km} / \mathrm{s}$ to $60 \mathrm{~km} / \mathrm{s}$, and 1 year mapping to $1 \mathrm{~ns},{ }^{29}$ where these values correspond to the dynamics occurring at times of 13 years in the SNR and $8 \mathrm{~ns}$ in the laboratory experiment. Once the shock impacts the ring, the shock transmitted into the ring may well be radiative, due to the much higher density. Then the simple Euler scaling will have to be modified.

Another well-known remnant, SN1006 [Fig. 4(a)], is a good example of how shock wave analysis techniques applied to recent images provide insights into the supernova that exploded in the year 1006 at a distance of $2 \mathrm{kpc} .{ }^{34}$ Spectral analysis of shock induced astrophysical emissions can yield the temperature, degree of equilibration, ionization state, and velocity of the shock. With an additional measure of the proper motion of the shock, the distance to the emitting source can also be determined. Such analysis of the shock-induced emissions from the remnant of SN1006 is given in Fig. 4(b). Here, emission lines from hydrogen (Ly$\operatorname{man} \beta$ ) and from 5-times-ionized oxygen (O VI) are identified. This is an example of emissions from a "nonradiative", 


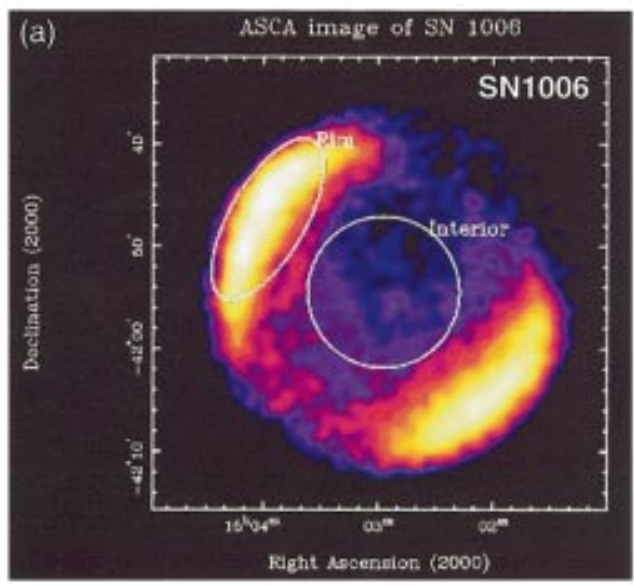

(b)

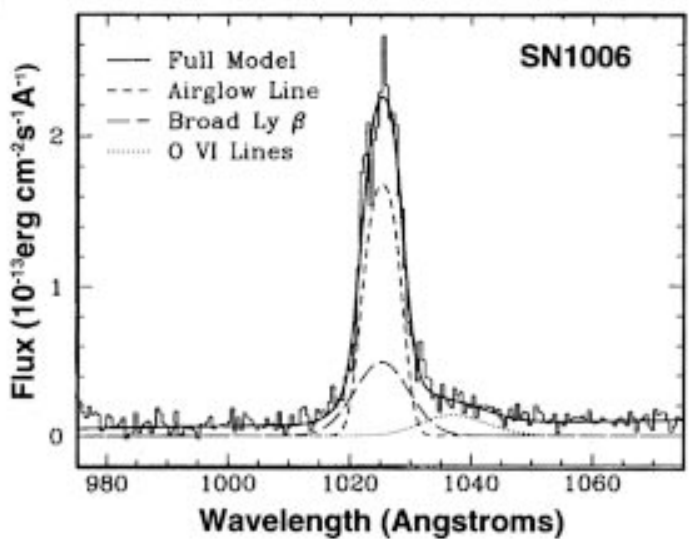

Laser experiment

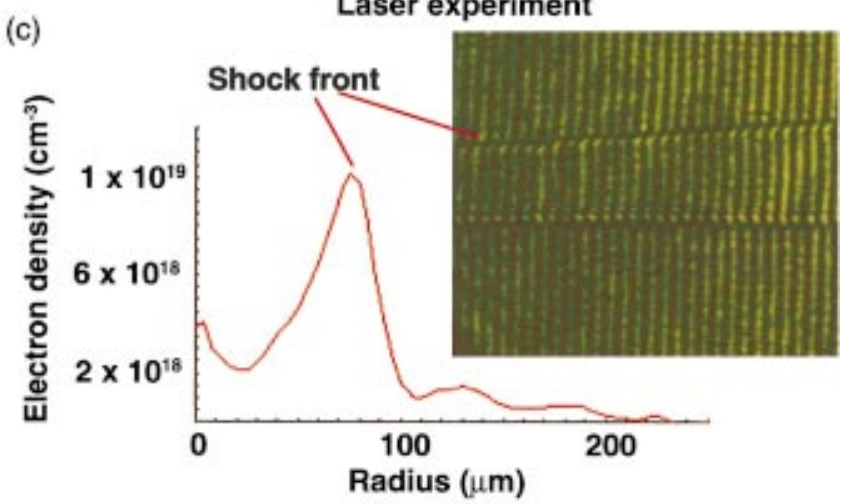

(d)

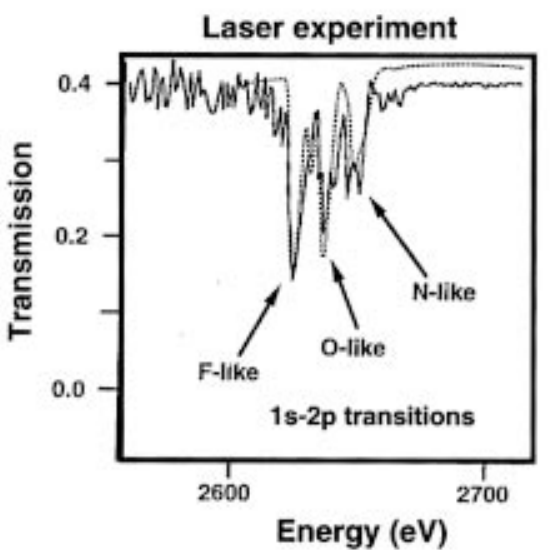

FIG. 4. (Color) Shocks in older supernova remnants. (a) Observational image of SN1006 (Ref. 34 and http://www-cr.scphys.kyoto-u.ac.jp/research/pix/ sn1006_i.gif). (b) Spectral analysis of the shocks from SN1006 (reproduced from Ref. 34). (c) Experimental image of a shock launched by a 40 fs, 15 mJ laser pulse in a gas cluster target, and diagnosed by optical interometry (Ref. 49). (d) Spectral analysis of experimentally generated shocks in foam targets, from which the temperature behind shock front can be determined (reproduced from Refs. 53 and 54).

shock-a shock traversing a low-enough density medium that the plasma behind the shock front is not cooling rapidly by radiation, $\tau_{\text {rad }} / \tau_{\text {hydro }} \gg 1$. The conclusion from this spectral analysis is that plasma turbulence in the shock front is not effective in producing temperature equilibration among the different ion species.

A long-standing mystery regarding astrophysical shocks is whether or not the electrons are strongly heated by the magnetized turbulence at the shock fronts of SNRs. ${ }^{45}$ The impact of electrons upon ions dominates the production of some emission lines, while the impact of protons and $\alpha$-particles dominates other (lower threshold) emission lines. $^{34}$ In the case of the shocks of the remnant from SN1006 and in several other cases analyzed to date, the electron temperature is found to be considerably below the ion temperature, $T_{e}=T_{i} / 4$, for a Mach-50 shock. ${ }^{34}$ This provides evidence that the magnetized turbulence at the shock wave does not rapidly force equilibration of the electrons and the ions. Developing an experimental setting to check the theories and analysis techniques of astrophysical shocks seems possible, at least for some situations, and would be very beneficial.

Laser-based experiments can produce strong shock waves for study in a variety of ways. In an experiment using a table-top laser, a gas jet target produces an assembly of clusters of atoms of order $10 \mathrm{~nm}$ in size, each containing thousands of atoms. These clusters absorb the laser radiation from a $800 \mathrm{~nm}$ Ti:Sapphire laser with a pulse duration of 0.1 ps or less. This produces intense heating, disassembly of the clusters ("a Coulomb explosion'), and radial expansion of the $2 \mathrm{~mm}$ long by initially $50 \mu \mathrm{m}$ diameter laser-irradiated hot, cylindrical channel. ${ }^{46-48}$ Under sufficiently collisional conditions [Fig. 4(c)], a Mach $\geqslant 10$ shock wave forms that drives the surrounding gas outward. ${ }^{49}$ Whether a magnetized shock can also be produced by this technique remains a topic for ongoing research. ${ }^{50}$ Given the high initial temperature of the shock, and the ability to experimentally vary the density and gas species, creating radiative and nonradiative shocks should be possible by this technique.

In astrophysical systems and the laboratory, shocks can 

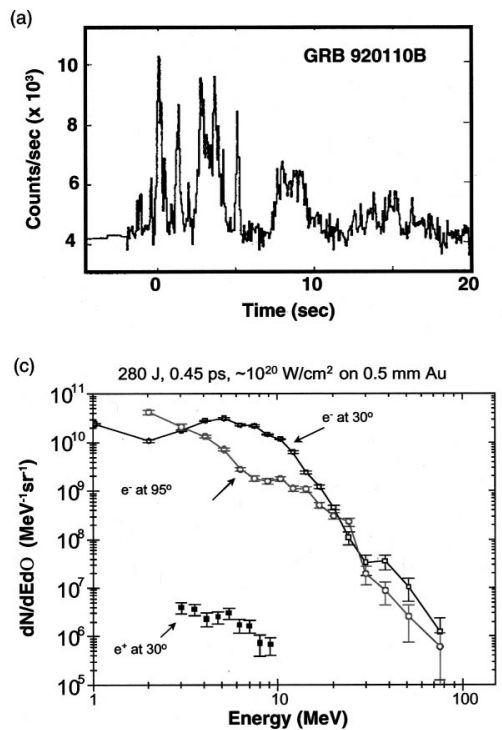
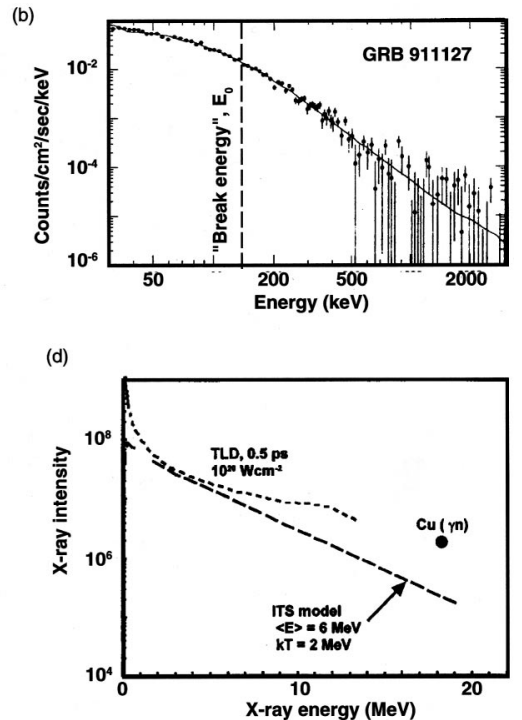

FIG. 5. Gamma ray bursts and relativistic plasmas. (a) Time evolution of the photon burst detected from GRB920110 (reproduced from Ref. 56). (b) Experimental $\gamma$-ray energy spectrum from GRB910601 (reproduced from Ref. 60). (c) Measured electron energy spectrum from Petawatt laser experiments (reproduced from Ref. 65). (d) Measured x-ray energy spectrum from experiments using the Petawatt laser (reproduced from Ref. 63). be produced for which radiation is essential to the dynamics. Radiation from the shock wave can cause preheat, altering the conditions ahead of the shock wave, and the shockgenerated radiation can be an important component of the energy flow within the system. An example from astrophysics is SN1993J, whose progenitor was a red supergiant star, surrounded by a very dense stellar wind. ${ }^{51}$ The resulting shock structure was strongly radiative and this affected the subsequent shock dynamics, leading to significantly higher densities behind the shock front. Laboratory shocks in which radiation affects the dynamics can also be created and studied. An experiment in which low-density foam was directly illuminated with an intense laser has been carried out, ${ }^{52}$ generating shocks whose radiation affected the matter ahead of them. Another radiative shock experiment ${ }^{53,54}$ has been conducted where the shock was launched by irradiating a foam target with soft $\mathrm{x}$-rays rather than direct laser illumination. [The foam used was $50 \mathrm{mg} / \mathrm{cm}^{3}$ triacrylate foam $\left(\mathrm{C}_{15} \mathrm{H}_{20} \mathrm{O}_{6}\right)$ chemically doped with a chlorine monomer $\left(\mathrm{C}_{9} \mathrm{H}_{3} \mathrm{O}_{2} \mathrm{Cl}_{5}\right)$ to $25 \%$ by weight chlorine.] Here, in three separate experiments, conditions were created corresponding to (1) pure hydrodynamics (subsonic radiation wave), (2) pure radiation flow (supersonic radiative wave), and (3) intermediate dynamics ("transonic"' regime). For each case, spectroscopy was used to determine the temperature profile in the plasma. For the case of the subsonic radiation front, the temperature behind the shock was determined spectroscopically, as shown in Fig. 4(d). What remains is to develop the theoretical transformation, mapping the conditions of astrophysical radiative shocks such as those associated with the remnant of SN1993J ${ }^{51}$ to the laboratory, so that laser experiments can be developed that are scaled reproductions of their astrophysical counterparts.

\section{GAMMA RAY BURSTS}

Gamma-ray bursts (GRB) are the greatest enigma in contempory astrophysics. ${ }^{55-59}$ Detected at a rate of more than one per day from random directions in the sky, GRBs typically have burst durations of a few seconds at photon energies of 0.1-10 MeV [Figs. 5(a) and 5(b)]. GRB distances remained unknown for the past two decades, primarily because their radiation in all other wavelengths other than $\gamma$-rays was undetected. This changed recently with the determination of accurate positions, obtained within hours of outburst by the BeppoSAX satellite. Optical spectroscopy of the " afterglow', associated with the GRB has revealed absorption and emission lines, giving recession velocities and the first conclusive determinations of distances to GRBs or their host galaxies. This has established that at least some of the GRBs are at cosmological distances of several billion light years (red shifts of $\Delta \lambda / \lambda=1$ to 3 ). To generate the observed luminosities then requires total source energies of $10^{51}-10^{53} \mathrm{ergs} /$ burst over $\sim 1-10 \mathrm{~s}$. The rapid rise time and rapid variability, $\Delta t \sim 1 \mathrm{~ms}$, observed in some bursts [Fig. 5(a) GRB 920110B] imply a source size, $R_{i} \sim c \Delta t$ $\sim 10^{7}-10^{8} \mathrm{~cm}$, i.e., these tremendous total energies appear to be emitted from very compact sources.

The observed photon energy spectra of a GRB can extend to $\sim 100 \mathrm{MeV}$ and typically exhibits a "low-energy", component $\left(E<E_{0}\right)$ and a high energy component $(E$ $>E_{0}$ ), the dividing energy $E_{0}$ being called the "break energy.' GRBs are often parameterized by fitting the energy spectra with the "Band function,", 60

$$
\begin{aligned}
& N_{E}(E) \sim E^{\alpha} e^{-E / E_{0}}, \text { for } E<E_{0}, \\
& N_{E}(E) \sim E^{\beta}, \text { for } E>E_{0} .
\end{aligned}
$$

An example spectrum fitted with Eq. (3) is illustrated for GRB911127 in Fig. 5(b), where the fit parameters are $\alpha=-0.97, \beta=-2.4$, and $E_{0}=150 \mathrm{keV}$. Large groups of GRBs can be categorized by their "Band parameters," which proves useful for testing various models. The fact that GRB spectra have a power law shape (as opposed to Planckian) is often interpreted as suggesting that the source plasma is optically thin to the radiation observed. [There are other models that allow an optically thick source. ${ }^{61}$ For the brief discussion here, we will assume an optically thin source, which is the more common assumption.] This presents a problem. When two photons with energies $E_{1}$ and $E_{2}$ inter- 
act, their center-of-mass energy is $\sim 2\left(E_{1} E_{2}\right)^{1 / 2}$, and the interaction can produce ${ }^{55}$ an $e^{+} e^{-}$pair if $\left(E_{1} E_{2}\right)^{1 / 2}>m_{e} c^{2}$. Denote the fraction of photon pairs in a GRB satisfying this condition as $f_{p}$. The optical depth (OD) for the $\gamma \gamma$ $\rightarrow e^{+} e^{-}$process varies as $\mathrm{OD} \sim f_{p} / R_{i}^{2}$, and is very large for typical GRB conditions. These $e^{+} e^{-}$pairs are produced prodigiously, and through Compton scattering, they would make the plasma optically thick, thermalizing the photon spectrum. The observed spectra appear nonthermal, however, whence the "compactness problem."

The fireball model was developed to resolve this problem without introducing " $n e w$ physics.", 55,58 Here, an initial release of $\sim 10^{52} \mathrm{ergs}$ of energy into a volume of spatial extent $\sim 10^{7} \mathrm{~cm}$ (by a mechanism not yet understood) creates a relativistically hot fireball of photons and leptons, with a small admixture of baryons. The initial temperature is given by

$$
T=(1 \mathrm{MeV})\left[\frac{E}{10^{50} \mathrm{ergs}}\right]^{1 / 4}\left[\frac{R_{i}}{10^{3} \mathrm{~km}}\right]^{-3 / 4},
$$

which, for typical parameters of $E=10^{51}-10^{53} \mathrm{ergs}$ and $R_{I}$ $=10^{3} \mathrm{~km}$, gives $T=2-6 \mathrm{MeV}$. If there were no baryons, the fireball would expand due to its own internal pressure, reaching highly relativistic velocities. Eventually, the fireball would become optically thin, at which point it would radiate profusely and cool. The observed photons would bear the spectral shape they had the moment the fireball became optically thin, and would appear thermal (Planckian). With a small admixture of baryons, however, the situation can be very different. The lepton component $\left(e^{+} e^{-}\right)$of the fireball expands initially much more rapidly than the baryon component, due to the much lower mass/particle. This creates an electric field that drags the baryons along. Under appropriate conditions, a large fraction of the initial energy content of the fireball can be transferred to the kinetic energy of the baryons, which also reach highly relativistic velocities. As the fireball sweeps outward into the interstellar medium ISM, a forward ("external") shock is launched into the ISM and a series of reverse ("internal") shocks are created in the fireball ejecta. This shock system resembles that of a SNR (see Sec. III), and, within the fireball model, GRBs can be thought of as relativistic SNRs. ${ }^{55,56}$ From the perspective of an observer at rest in the "lab frame," consider such a forward shock-reverse shock system where the shock velocities are relativistic. The expanding cloud of baryons are assumed to transfer energy to electrons by the collisionless shock mechanisms, ${ }^{62}$ and the electrons are assumed to create photons by synchrotron radiation or by inverse Compton scattering. Hence, one has a source of radiation (the shocks) moving toward the observer at relativistic velocities, ${ }^{55,59}$ characterized by a Lorentz factor, $\Gamma=1 /\left(1-v^{2} / c^{2}\right)^{1 / 2} \gg 1$. The observer detects photons with energy $h v_{\text {obs }}$ whereas in the rest frame of the emission region, these photons have a much lower energy $h v_{\text {obs }} / \Gamma$. In the frame of the emitter, the fraction of photons with energies high enough to produce $e^{+} e^{-}$pairs, $f_{p}$, is now reduced by a factor $\Gamma^{2 \alpha}$. Also, due to the high velocity of the source $(1-v / c \ll 1)$, the characteristic time scales in the frame of the source will be a factor of
$2 \Gamma^{2}$ longer, ${ }^{55,61}$ giving $R_{i} \sim \Gamma^{2} c \Delta t$. The net result is that the optical depth for the process $\gamma \gamma \rightarrow e^{+} e^{-}$now varies as OD $\sim f_{p} / \Gamma^{4+2 \alpha} R_{i}^{2}$, which for $\Gamma>\sim 100$ resolves the compactness problem. Through the blue shift boost, we observe the high energy photons, but the emission region remains optically thin, giving the observed $\gamma$-ray power-law spectrum. The kinetic energy of the GRB ejecta is randomized behind reverse shocks, and emitted as high energy photons when the shock is at a radius of $R_{\mathrm{int}}=\Gamma^{2} c \Delta t=10^{12}-10^{13} \mathrm{~cm}$, for $\Gamma$ $=100-300$. The "afterglow" results from emissions behind the forward shock at a radius of $R_{\text {ext }}>\sim 10^{17} \mathrm{~cm}$.

In summary, an initial fireball of leptons and photons at an initial temperature of $T \sim 1-10 \mathrm{MeV}$ expands relativistically. This accelerates a small admixture of baryons to relativistic velocities, thereby transferring the fireball initial thermal energy to the kinetic energy of the radially expanding baryons. The baryons expand into the ISM, creating a system of forward shock and several reverse shocks, with the observed GRB emission coming from the reverse shocks. The afterglow then comes from the forward shock. This can be thought of as a 4-step process: (1) a source or "engine", creates the initial radiation-lepton fireball; (2) the lepton fireball thermal energy is transferred to the directed kinetic energy of baryons; (3) the baron expansion into the ISM generates a forward shock-reverse shocks system; and (4) the shocks randomize the baryon kinetic energy, which transfers energy to photons. The overall expansion by this time is large, the plasma is optically thin, and the photons, once created, escape.

Aspects of the underlying fireball physics may be accessible in the lab. As an example, we consider experiments that were done with an ultra-high intensity laser at powers reaching a petawatt. ${ }^{63,64}$ In these experiments, the laser pulses of $\sim 500 \mathrm{~J}$ of energy at $\lambda=1 \mu \mathrm{m}$ wavelength in $0.5-5 \mathrm{ps}$ pulses in $\mathrm{a} \sim 10 \mu \mathrm{m}$ spot, gave laser intensities of $\sim 10^{20} \mathrm{~W} / \mathrm{cm}^{2}$. The interaction of this pulse with the target leads to heating of electrons (leptons) to relativistic temperatures equivalent roughly to the ponderomotive potential of the laser beam (that is, the cycle-averaged kinetic energy of an electron oscillating in the laser electromagnetic field). The relativistic electron temperature is given by ${ }^{64}$

$$
T_{\text {hot }}=(1 \mathrm{MeV})\left[\frac{I \lambda^{2}}{10^{19} \mathrm{~W} \mathrm{~cm}^{-2} \mu \mathrm{m}^{2}}\right]^{1 / 2} \text {. }
$$

The experiments were carried out at a range of intensities, peaking at $\sim 3 \times 10^{20} \mathrm{~W} / \mathrm{cm}^{2}$, corresponding to "laboratory fireball' temperatures of $1-5 \mathrm{MeV}$. These initial temperatures are intriguingly similar in magnitude to the initial conditions of the GRB lepton fireball [see Eq. (4)], i.e., "Step 1"' in GRB generation. A typical measured electron and positron energy spectrum ${ }^{65}$ is shown in Fig. 5(c), showing a broad peak at $5 \mathrm{MeV}$ and energies extending up to 100 $\mathrm{MeV}$. The measured and simulated bremsstrahlung spectrum is shown in Fig. 5(d). Fitting the bremsstrahlung data with an $I_{0} e^{-h \nu / T}$ functional form gives an exponential "temperature", of $T_{\gamma} \approx 4 \mathrm{MeV}$, consistent with $T_{\gamma} \approx T_{\text {hot }}$ given by Eq. (5). It is estimated that $40 \%-50 \%$ of the initial laser energy was converted to these hot electrons, positrons, and photons, gen- 


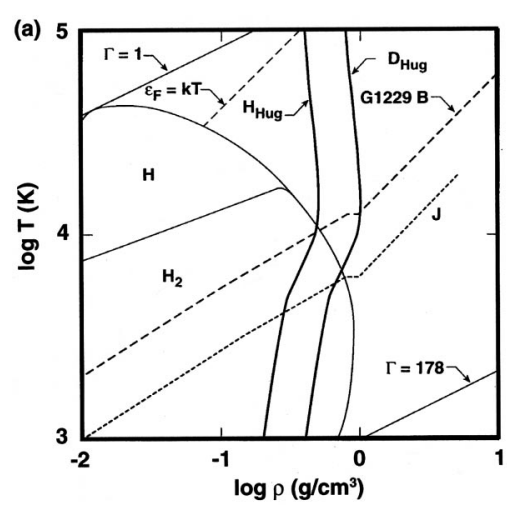

(b)

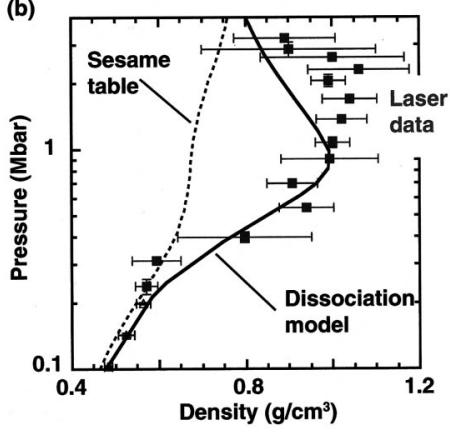

FIG. 6. The phase diagram and equation-of-state (EOS) experiments relevant to the giant planets and brown dwarfs. (a) Theoretical phase diagram of hydrogen (reproduced from Ref. 77) relevant to Jupiter and the brown dwarf G1229B. (b) Measured compression (density) versus shock-induced pressure, that is, the measured principle Hugoniot for cryogenic liquid $\mathrm{D}_{2}$ (reproduced from Ref. 77). erating a laboratory fireball of leptons and radiation. [High energy $(>10 \mathrm{MeV})$ bremsstrahlung photons have also been observed at $T_{\gamma} \approx 1.2 \mathrm{MeV}$ in ultraintense laser experiments $\left(I_{\text {Laser }}=10^{19} \mathrm{~W} / \mathrm{cm}^{2}\right)$ at other facilities, suggesting that their production is a general result of intense laser-matter interactions. $]^{66}$

Another intriguing observations in the petawatt experiments was the generation of luminous beams of protons (baryons) from the rear of the target. ${ }^{64,67}$ Proton energies up to at least $55 \mathrm{MeV}$ were observed, and it is estimated that $6 \%$ of the initial laser energy ended up in this energetic, bright proton beam. The mechanism proposed for generating the proton beam is acceleration by a collective electrostatic (sheath) field generated by the hot electrons. Only a very small fraction of the hot electrons created in the laser-target interaction can leave the target before the resulting Coulomb potential traps the rest. The trapped hot electrons create a sheath at the target surfaces with a scale length given by the Debye length $\left(l_{\mathrm{De}}\right)$ of the hot electrons. This leads to a very strong sheath electrostatic field, ${ }^{64,68} E_{\text {field }} \approx k T_{\text {hot }} /\left(e l_{\text {De }}\right)$, which gives enormous field strengths of order $\mathrm{MV} / \mu \mathrm{m}$ that accelerate the proton jets. This proton acceleration continues until the hot electrons are energetically depleted by transferring their energy to the protons. The proton energy $\left(\epsilon_{p}\right)$ scales as $\epsilon_{p} \sim E_{\text {field }}^{2} \sim($ laser energy). Ion jets have been observed in short-pulse, high intensity laser experiments at other facilities as well, suggesting that ion jet generation is a common feature of laser-target interactions at very high intensity. ${ }^{69,70}$ Strong magnetic field generation ( $>1$ Mgauss) has also been observed in ultraintense laser experiments, ${ }^{71}$ with simulations predicting fields stronger yet $(>100$ Mgauss). ${ }^{72}$

In GRB terminology, the initial hot lepton (electron) fireball transfers its energy to the kinetic energy of the baryons (proton jet), which are accelerated to high velocity, similar to "Step 2"' within the GRB fireball model. The laser experiment baryon jet was not relativistic, but on future lasers with more energy, perhaps they could be. Furthermore, if the target had had a low density ambient gas or foam, this baryon jet would have launched a forward shock and reverse shock, adding similarity to "Step 3"' within the GRB fireball model.

\section{GIANT PLANETS}

The "high stakes tug of war' between quantum mechanical degeneracy pressure and the more familiar gravitational pressure was discussed above in Sec. II. A somewhat more benign environment to consider strong degeneracy effects is in the steady state interiors of the giant planets such as Saturn and Jupiter and the newly discovered brown dwarfs, ${ }^{7,73-76}$ as represented by the phase diagram ${ }^{77}$ shown in Fig. 6(a). Here, because of their lower mass, $M$ $\leqslant 0.08 M_{\text {sun }}$, these bodies never ignite as stars, and the degeneracy pressure and strongly coupled effects dominate.

Strongly coupled plasmas are typically characterized by the dimensionless parameter, $\Gamma=(Z e)^{2} / a k T$, where $a$ is a characteristic separation distance between ions. In plasmas with $\Gamma \ll 1$, thermal effects dominate and the plasma is considered "ideal." When $\Gamma \geqslant 1$, the Coulomb interactions become an equal player, and the plasma enters the strongly coupled regime, represented by the region to the right and below the $\Gamma=1$ line in Fig. 6(a). When $\Gamma>178$, the plasma becomes so strongly coupled that the ions freeze solid into a crystal lattice. Also, when the densities are high enough or temperatures low enough that $k T<\epsilon_{F}$, where $\epsilon_{F}=p_{F}^{2} / 2 m_{e}$ $=(1 / 8)(3 / \pi)^{2 / 3}\left(h^{2} / m_{e}\right) n_{i}^{2 / 3} \propto \rho^{2 / 3}$ is the Fermi energy, the plasma is called degenerate, and is represented by the region to the right and below the $\epsilon_{F}=k T$ line in Fig. 6(a). Here, electron degeneracy pressure becomes a major part of the total pressure. The isentropes for Jupiter and the brown giant G1229B ${ }^{75}$ shown in Fig. 6(a) indicate that these bodies, which are made up predominantly of $\mathrm{H}$ and $\mathrm{He},{ }^{76}$ are both strongly coupled and highly degenerate. Hence, the internal structure, $\rho(r), T(r)$, and to some extent the external magnetic fields of the giant planets are determined by the EOS of degenerate hydrogen and helium at high pressure, $P$ $=1-100$ Mbar. The EOS of strongly coupled, degenerate plasma, however, is notoriously difficult to calculate from first-principles theories, due to the complexity of including quantum mechanical effects into classical thermodynamic theories. Experiments in this parameter regime are a vital component in efforts to improve our understanding of Jupiter, the other giant planets, and brown dwarfs.

The EOS of a material can be determined by measuring its response to a known applied pressure. Measurements of 
the EOS of cryogenic deuterium, D (an isotope of hydrogen) at applied pressures ranging from $220 \mathrm{kbar}$ to 3.4 Mbar have been made on recent laser experiments. ${ }^{77}$ In these experiments, the transition of hydrogen from a molecular fluid insulator phase to a monatomic metallic phase was unambiguously observed. A clear departure from the standard theoretical EOS models for hydrogen was found in the compressibility of $D_{2}$ in this regime [Fig. 6(b)]. The results were consistent with a new model that included the potential energy sink caused by molecular dissociation $\left(D_{2} \rightarrow D+D\right)$. These results, together with extensive results form gas-gun experiments at lower pressure, ${ }^{78,79}$ have implications for the composition and dynamics of the outer layers of Jupiter, the other giant planets, and brown dwarfs.

The pressure and temperature in the mantle of Jupiter near the surface are in the range of 1-3 Mbar and less than of an $\mathrm{eV}$. Deeper in the interior, the pressure and temperature increase, rising to $40 \mathrm{Mbar}$ and a couple of $\mathrm{eV}$ at the center ${ }^{80}$ Near the surface, hydrogen exists as the molecule $\mathrm{H}_{2}$, but dissociates to $\mathrm{H}+\mathrm{H}$ and ionizes deeper in the mantle. This transition of hydrogen from insulator to conductor is important because conducting $\mathrm{H}$ in the convective zone is thought to create the 10-15 Gauss magnetic field of Jupiter. ${ }^{81}$ One of the fundamental open questions about the interior of Jupiter is whether there is a sharp boundary, a plasma phase transition (PPT), between a molecular hydrogen mantle and a monatomic hydrogen core at a radius of $\sim 0.75 R_{J}$ and pressure of $3 \mathrm{Mbar}$. The regimes accessed by the laser and gas-gun experiments represented on Fig. 6(b) span this critical transition from mantle to core of Jupiter, and suggest that a sharp discontinuity between molecular (mantle of Jupiter) to monatomic (core of Jupiter) hydrogen does not exist. The experiments ${ }^{77,81}$ suggest that on the Jovian isentrope molecular hydrogen probably begins to dissociate at $400 \mathrm{kbar}$ and dissociation continues smoothly to completion at $\sim 3 \mathrm{Mbar}$, with metallization occurring right in the middle of this region at $\sim 1.4 \mathrm{Mbar}$ and $\sim 4000 \mathrm{~K}$. It now seems likely ${ }^{81}$ that currents near the surface of Jupiter, at radii out to $0.95 R_{J}$ contribute to the surface magnetic field, whereas previously it was thought that the magnetic field was formed deeper in the interior at $\sim 0.75 R_{J}$. The EOS of astrophysically relevant materials are being measured on several other laser facilities as well. ${ }^{82-87}$ This whole area of measuring high pressure EOS on intense lasers represents fertile new territory for planetary and astrophysics research.

Finally, we mention in closing that descriptions of the structure of white dwarfs have been a long standing theoretical challenge ${ }^{88}$ Although conditions in the interior are probably not within experimental reach in the foreseeable future, the outer atmospheres are not so physically remote. Typical values for temperature, density, and pressure for a carbon white dwarf atmosphere ${ }^{89}$ range from $10^{5} \mathrm{~K}, 1 \mathrm{~g} / \mathrm{cm}^{3}, 2$ Mbar to $10^{6} \mathrm{k}, 10^{2} \mathrm{~g} / \mathrm{cm}^{3}, 1$ Gbar, respectively. An understanding of white dwarf atmospheres is important because the atmosphere determines the cooling rate. These conditions are extreme, to be sure, but fall within the realm of experiments being planned for the next generation "superlasers" currently under construction. ${ }^{90,91}$

\section{ACKNOWLEDGMENT}

The work was carried out under the auspices of the U.S. Department of Energy by the Lawrence Livermore National Laboratory under contract number W-7405-ENG-48. We are indebted to the many scientists who generously shared their data and thoughts with us as we composed this review. We offer a special note of gratitude and thanks to Kimberly D. Morris for her skilled and conscientious assistance in all phases of the preparation of this manuscript.

${ }^{1}$ B. A. Remington, D. Arnett, R. P. Drake, and H. Takabe, Science 284, 1488 (1999).

${ }^{2}$ W. D. Arnett, J. N. Bahcall, R. P. Kershner, S. E. Woolsey, Annu. Rev. Astron. Astrophys. 27, 629-700 (1989).

${ }^{3}$ W. Hillebrandt and P. Höflich, Rep. Prog. Phys. 52, 1421 (1989).

${ }^{4}$ D. Arnett, Supernovae and Nucleosynthesis (Princeton Univ. Press, Princeton, NJ, 1996).

${ }^{5}$ H. Bethe, Rev. Mod. Phys. 62, 801 (1990).

${ }^{6}$ S. Woosley and T. Weaver, Sci. Am. 261(8), 32 (Aug. 1989).

${ }^{7}$ H. M. Van Horn, Science 252, 384 (1991).

${ }^{8}$ P. K. Shukla, L. O. Silva, H. Bethe, R. Bingham, J. M. Dawson, L. Stenflo, J. T. Mendonca, and S. Dalhed, Plasma Phys. Controlled Fusion 41, A699 (1999); L. O. Silva, R. Bingham, J. M. Dawson, J. T. Mendonca, and P. K. Shukla, Phys. Rev. Lett. 83, 2703 (1999).

${ }^{9}$ D. Arnett, "Hydrodynamic Mixing and Nucleosynthesis," Astrophy. J. Suppl. (to be published).

${ }^{10}$ W. D. Arnett, Astrophys. J. 331, 377 (1988).

${ }^{11}$ L. Ensman and A. Burrows, Astrophys. J. 393, 742 (1992).

${ }^{12}$ B. P. Schmidt, R. P. Kirshner, and R. G. Eastman, Astrophys. J. 395, 366 (1992); ibid. 432, 42 (1994).

${ }^{13}$ R. G. Eastman, B. P. Schmidt, and R. Kirshner, Astrophys. J. 466, 911 (1996); ibid. 430, 300 (1994).

${ }^{14}$ D. Branch, E. Baron, P. Nugent, and P. H. Hauschildt, Phys. Plasmas 4, 2016 (1997).

${ }^{15}$ C. A. Iglesias and F. J. Rogers, Astrophys. J. 464, 943 (1996).

${ }^{16}$ P. T. Springer et al., Phys. Rev. Lett. 69, 3735 (1992).

${ }^{17}$ J. M. Foster et al., Phys. Rev. Lett. 67, 3255 (1991).

${ }^{18}$ T. S. Perry et al., Phys. Rev. Lett. 67, 3784 (1991).

${ }^{19}$ L. B. Da Silva et al., Phys. Rev. Lett. 69, 438 (1992).

${ }^{20}$ K. J. Kearney, A. N. Mostovych, and J. A. Stamper, Rev. Sci. Instrum. 61, 3596 (1990)

${ }^{21}$ C. A. Back et al., J. Quant. Spectrosc. Radiat. Transf. 58, 415 (1997).

${ }^{22}$ C. Chenais-Popovics, H. Merdji, T. Missalla, F. Gilleron, J-C. Gauthier, T. Blenski, F. Perrot, M. Klapisch, C. Bauche-Arnoult, J. Bauche, A. Bachelier, and K. Eidmann, "Opacity studies on iron in the 15-30 eV temperature range," Astrophy. J. Suppl. (to be published).

${ }^{23}$ P. T. Springer, K. L. Wong, C. A. Iglesia, J. H. Hammer, J. L. Porter, A. Toor, W. H. Goldstein, B. G. Wilson, F. J. Rogers, C. Deeney, D. S. Dearborn, C. Bruns, J. Emig, and R. E. Stewart, J. Quant. Spectrosc. Radiat. Transf. 58, 927 (1997).

${ }^{24}$ J. Wark, S. J. Rose, P. K. Patel, A. Djaoui, O. Renner, and A. Hauer, Phys. Plasmas 4, 2004 (1997); Phys. Rev. Lett. 75, 1680 (1994); P. K. Patel, E. Wolfrum, O. Renner, A. Loveridge, R. Alllot, D. Neely, S. J. Rose, and J. S. Wark, J. Quant. Spectros. Radiat. Transfer 65, 429 (1999).

${ }^{25}$ E. Muller, B. Fryxell, and D. Arnett, Astron. Astrophys. 251, 505 (1991).

${ }^{26}$ B. Fryxell, E. Muller, and D. Arnett, Astrophys. J. 367, 619 (1991).

${ }^{27}$ J. Kane, D. Arnett, B. A. Remington, S. G. Glendinning, J. Castor, R. J. Wallace, A. Rubenchik, and B. A. Fryxell, Astrophys. J. 478, L75 (1997); J. Kane, D. Arnett, B. A. Remington, S. G. Glendinning, D. Ryutov, B. A. Fryxell, R. P. Drake, K. Moore, R. Teyssier, Phys. Plasmas 6, 2065 (1999).

${ }^{28}$ B. A. Remington, J. O. Kane, R. P. Drake, S. G. Glendinning, K. Estabrook, R. London, J. Castor, R. J. Wallace, D. Arnett, E. Liang, R. McCray, A. Rubenchik, and B. Fryxell, Phys. Plasmas 4, 1994 (1997).

${ }^{29}$ D. D. Ryutov, R. P. Drake, J. O. Kane, E. Liang, B. A. Remington, and W. M. Wood-Vasey, Astrophys. J. 518, 821 (1999).

${ }^{30}$ M. M. Marinak, B. A. Reemington, S. V. Weber, R. E. Tipton, S. W. Haan, K. S. Budil, O. L. Landen, J. D. Kilkenny, and R. J. Wallace, Phys. Rev. Lett. 75, 3677 (1995).

${ }^{31}$ U. Hwang, J. P. Hughes, and R. Petre, Astrophys. J. 497, 833 (1998); P. F. 
Velazquez, D. O. Gomez, G. M. Dubner, G. Gimenez de Castro, and A. Costa, Astron. Astrophys. 334, 1060 (1998).

${ }^{32}$ K. J. Borkowski and A. E. Szymkowiak, Astrophys. J. 477, L49 (1997); R. Braun, Astron. Astrophys. 171, 233 (1987); E. Dwek, Astrophys. J. 322, 812 (1987)

${ }^{33}$ N. A. Levenson, J. R. Graham, J. D. Keller, and M. J. Richter, Astrophys. J., Suppl. 118, 541 (1998); Astrophys. J. 484, 304 (1997); ibid. 468, 323 (1996); J. J. Hester, J. C. Raymond, and W. P. Blair, Astrophys. J. 420, 721 (1994); R. A. Resen, K. B. Kwitter, and R. A. Downes, Astrophys. J. 104, 719 (1992).

${ }^{34}$ J. C. Raymond, W. P. Blair, and K. S. Long, Astrophys. J. 454, L31 (1995); P. F. Winkler and K. S. Long, Astrophys. J. 486, L137 (1997); ibid. 491, 829 (1997); J. M. Laming, J. C. Raymond, B. M. McLaughlin, and W. P. Blair, Astrophys. J. 472, 267 (1996); K. Koyama, R. Petre, E. V. Gotthelf, U. Hwang, M. Matsuura, M. Ozaki, and S. S. Holt, Nature 378, 255 (1995).

${ }^{35}$ J. Hester, J. M. Stone, P. A. Scowen, B. I. Jun, J. S. Gallagher III, M. L. Norman, G. E. Ballester, C. J. Burrows, S. Casertano, J. T. Clarke, D. Crisp, R. E. Griffiths, J. G. Hoessel, J. A. Holtzman, J. Krist, J. R. Mould, R. Sandrit, K. R. Stapelfeld, J. T. Trauger, A. Watson, and J. A. Westphal, Astrophys. J. 456, 225 (1996).

${ }^{36}$ K. J. Borkowski, J. M. Blondin, and R. McCray, Astrophys. J. 477, 281 (1997).

${ }^{37}$ K. J. Borkowski, J. M. Blondin, and R. McCray, Astrophys. J. 476, L31 (1997).

${ }^{38}$ E. Michael, R. McCray, K. J. Borkowski, C. S. J. Pun, and G. Sonneborn, Astrophys. J. 492, L143 (1998); ibid. 509, L117 (1998).

${ }^{39}$ R. P. Drake, J. J. Carroll III, K. Estabrook, S. G. Glendinning, B. A. Remington, R. J. Wallace, and R. McCray, Astrophys. J. Lett. 500, L157 (1998).

${ }^{40}$ R. P. Drake, S. G. Glendinning, K. Estabrook, B. A. Remington, R. McCray, R. J. Wallace, L. J. Suter, T. B. Smith, J. J. Carroll III, R. A. London, and E. Liang, Phys. Rev. Lett. 81, 2068 (1998).

${ }^{41}$ J. Kane, R. P. Drake, and B. A. Remington, Astrophys. J. 511, 335 (1999).

${ }^{42}$ R. A. Chevalier, Astrophys. J. 258, 790 (1982).

${ }^{43}$ R. I. Klein, T. S. Perry, K. S. Budil, and D. R. Bach, "The interaction of supernova remnants with interstellar clouds: from the Nova laser to the galaxy," Astrophy. J. Suppl. (to be published).

${ }^{44}$ R. I. Klein, C. F. McKee, and P. Colella, Astrophys. J. 420, 213 (1994).

${ }^{45}$ J. M. Laming, J. C. Raymond, B. M. McLaughlin, and W. P. Blair, Astrophys. J. 472, 267 (1996).

${ }^{46}$ T. Ditmire, J. W. G. Tisch, E. Springate, M. B. Mason, N. Hay, J. P. Marangos, and M. H. R. Hutchinson, Phys. Rev. Lett. 78, 2732 (1997); ibid. 78, 3121 (1997); Nature (London) 386, 54 (1997); T. Ditmire, K. Shigemori, B. A. Remington, K. Estabrook, and R. A. Smith, "The Production of Strong Blast Waves Through Intense Laser Irradiation of Atomic Clusters," Astrophy. J. Suppl. (to be published).

${ }^{47}$ G. S. Sarkisov, V. Y. Bychenkov, V. T. Tichonchuk, A. Maksimchuk, S. Y. Chen, R. Wagner, G. Mourou, and D. Umstadter, JETP Lett. 66, 828 (1997).

${ }^{48}$ S. Y. Chen, G. S. Sarkisov, A. Maksimchuk, R. Wagner, and D. Umstadter, Phys. Rev. Lett. 80, 2610 (1998).

${ }^{49}$ K. Shigemori, T. R. Ditmore, T. Kuhl, and B. A. Remington, Bull. Am. Phys. Soc. 43, 1731 (1998), paper F3S38; K. Shigemori, T. Ditmire, B. A. Remington, V. Yanovsky, D. Ryutov, K. G. Estabrook, M. J. Edwards, A. J. Mackinnon, A. M. Rubenchik, K. A. Keilty, and E. Liang, "Developing a radiative shock experiment relevant to astrophysics," Astrophy. J. Lett. (to be published)

${ }^{50}$ D. Ryutov, R. P. Drake, and B. A. Remington, "Criteria for scaled laboratory simulations of astrophysical MHD phenomena," Astrophy. J. Suppl. (to be published).

${ }^{51}$ R. A. Chevalier, Science 276, 1374 (1997); T. Suzuki and K. Nomoto, Astrophys. J. 455, 658 (1995); C. Fransson, P. Lundqvist, R. A. Chevalier, ibid. 461, 993 (1996); J. M. Marcaide, A. Alberdi, E. Ros, P. Diamond, I. I. Shapiro, J. C. Guirado, D. L. Jones, T. P. Krichbaum, F. Mantovani, R. A. Preston, A. Rius, R. T. Schillizzi, C. Trigilio, A. R. Whitney, and A. Witzel, Science 270, 1475 (1995); R. A. Chevalier and C. Fransson, Astrophys. J. 420, 268 (1994).

${ }^{52}$ J. C. Bozier, J. P. Le Breton, G. Thiell, S. Azra, M. Decroisette, and D. Schirmann, Phys. Rev. Lett. 57, 1304 (1986)

${ }^{53}$ T. Afshar-rad, M. Desselberger, M. Dunne, J. Edwards, J. M. Foster, D. Hoarty, M. W. Jones, S. J. Rose, P. A. Rosen, R. Taylor, and O. Willi, Phys. Rev. Lett. 73, 74 (1994).
${ }^{54}$ D. Hoarty, A. Iwase, C. Meyer, J. Edwards, and O. Willi, Phys. Rev. Lett. 78, 3322 (1997).

${ }^{55}$ T. Piran, in Unsolved Problems in Astrophysics, edited by John N. Bahcall and Jeremiah P. Ostriker (Princeton University Press, Princeton, New Jersey, 1997), pp. 343-369.

${ }^{56}$ G. J. Fishman and C. A. Megan, Annu. Rev. Astron. Astrophys. 33, 415 (1995); G. J. Fishman, C. A. Meegan, R. B. Wilson, M. N. Brock, J. M. Horack, C. Kouveliotou, S. Howard, W. S. Paciesas, M. S. Briggs, G. N. Pendleton, T. M. Koshut, and J. P. Lestrade, Astrophys. J. Suppl. 92, 229 (1994).

${ }^{57}$ E. Waxman, Astrophys. J. 489, L33 (1997).

${ }^{58} \mathrm{E}$. Waxman, "Gamma-ray bursts: afterglow, high energy cosmi-rays, and neutrinos," Astrophy. J. Suppl. (to be published).

${ }^{59}$ J. H. Krolick and E. A. Pier, Astrophys. J. 373, 277 (1991).

${ }^{60}$ D. Band, J. Matteson, L. Ford, Astrophys. J. 413, 281 (1993).

${ }^{61}$ S. I. Blinnikov, A. V. Kozyreva, and I. E. Panchenko, Astron. Rep. 43, 739 (1999).

${ }^{62}$ P. J. Cargill and K. Papadopoulos, Astrophys. J. 329, L29 (1988); A. R. Bell, Astrophys. Space Sci. 256, 13 (1998); A. R. Bell, P. Choi, A. E. Dangor, W. O. Willi, D. A. Bassett, C. J. Hooker, Phys. Rev. A 38, 1363 (1988); MNRAS 182, 147 (1978).

${ }^{63}$ M. H. Key, M. D. Cable, T. E. Cowan, K. G. Estabrook, B. A. Hammel, S. P. Hatchett, E. A. Henry, D. E. Hinkel, J. D. Kilkenny, J. A. Koch, W. L. Kruer, A. B. Langdon, B. F. Lasinski, R. W. Lee, B. J. MacGowan, A. Mackinnon, J. D. Moody, M. J. Moran, A. A. Offenberger, D. M. Pennington, M. D. Perry, T. J. Phillips, T. C. Sangster, M. S. Singh, M. A. Stoyer, M. Tabak, G. L. Tietbohl, M. Tsukamoto, K. Wharton, and S. C. Wilks, Phys. Plasmas 5, 1966 (1998).

${ }^{64}$ S. P. Hatchett, C. G. Brown, T. E. Cowan, Eugene A. Henry, Joy S. Johnson, Michael H. Key, Jeffrey A. Koch, A. Bruce Langdon, Barbara F. Lasinski, Richard W. Lee, Andrew J. Mackinnon, Deanna M. Pennington, Michael D. Perry, Thomas W. Phillips, Markus Roth, T. Craig Sangster, Mike S. Singh, Richard A. Snavely, Mark A. Stoyer, Scott C. Wilks, Kazuhito Yasuike, Phys. Plasmas 7, 2076 (2000).

${ }^{65} \mathrm{~T}$. Cowan, et al. in Proc. of XXIV ECLIM, to be published in Lasers Part. Beams (2000).

${ }^{66}$ P. A. Norreys, M. Santala, E. Clark, M. Zepf, I. Watts, F. N. Beg, K. Krushilnick, M. Tatarakis, A. E. Dangor, X. Fang, P. Graham, T. McCanny, R. P. Singhal, K. W. D. Ledingham, A. Creswell, D. C. W. Sanderson, J. Magill, A. Machacek, J. S. Wark, R. Allot, B. Kennedy, and D. Neely, Phys. Plasmas 6, 2150 (1999).

${ }^{67}$ R. Snavely, S. Hatchett, M. Key, C. Brown, T. Cowan, G. Henry, B. Langdon, B. Lasinski, A. MacKinnoiin, D. Pennington, M. Perry, T. Phillips, M. Roth, C. Sangster, M. Singh, M. Stoyer, S. Wilks, and K. Yasuike, Phys. Rev. Lett. (submitted); ibid. Bull. Am. Phys. Soc. 44, 229 (1999).

${ }^{68}$ J. Denevitt, Phys. Fluids 22, 1384 (1979).

${ }^{69}$ A. Maksimchuk, S. Gu, K. Flippo, and D. Umstadter, "Forward ion acceleration in thin films driven by a high intensity laser," Phys. Rev. Lett. (submitted).

${ }^{70}$ A. R. Bell, F. N. Beg, Z. Chang, A. E. Dangor, C. N. Danson, C. B. Edwards, A. P. Fews, M. H. R. Hutchinson, S. Laun, P. Lee, P. A. Norreys, R. A. Smith, P. F. Taday, and F. Zhou, Phys. Rev. E 48, 2087 (1993).

${ }^{71}$ M. Borghesi, A. J. MacKinnon, R. Gaillard, O. Willii, A. Pukhov, and J. Meyer-ter-Vehn, Phys. Rev. Lett. 80, 5137 (1998); Phys. Rev. Lett. 81, 112 (1998).

${ }^{72}$ A. Pukhov and J. Meyer-ter-Vehn, Phys. Plasmas 5, 1880 (1998); Phys. Rev. Lett. 76, 3975 (1996); B. Lasinski, A. B. Langdon, S. P. Hatchett, M. H. Key, and M. Tabak, Phys. Plasmas 6, 2041 (1999); S. C. Wilks, W. L. Kruer, M. Tabak, and A. B. Langdon, Phys. Rev. Lett. 69, 1383 (1992); Phys. Fluids B 5, 2603 (1993).

${ }^{73}$ W. B. Hubbard, T. Guillot, J. I. Lunine, A. Burrows, D. Saumon, M. S. Marley, and R. S. Freedman, Phys. Plasmas 4, 2011 (1997).

${ }^{74}$ W. R. Magro, D. M. Ceperley, C. Pierleoni, and B. Bernu, Phys. Rev. Lett. 76, 1240 (1996); D. Saumon, W. B. Hubbard, A. Burrows, T. Guillot, J. L. Lunine, and G. Chabrier, Astrophys. J. 460, 993 (1996).

${ }^{75}$ T. Nakajima, B. R. Oppenheimer, S. R. Kulkarni, D. A. Golimowski, K. Matthews, and S. T. Durrance, Nature (London) 378, 463 (1995); B. R. Oppenheimer, S. R. Kulkarni, K. Matthews, and T. Nakajima, Science 270, 1478 (1995).

${ }^{76}$ T. Encrenaz, Astron. Astrophys. Rev. 9, 171 (1999).

${ }^{77}$ G. W. Collins, L. B. DaSilva, P. Celliers, D. M. Gold, M. E. Foord, R. J. Wallace, A. Ng, S. V. Weber, K. S. Budil, and R. Cauble, Science 281, 1178 (1998); Phys. Plasmas 5, 1864 (1998); L. B. Da-Silva, P. Celliers, G. W. Collins, K. S. Budil, N. C. Holmes, T. W. Barbee, Jr., B. A. Hammel, 
J. D. Kilkenny, R. J. Wallace, M. Ross, R. Cauble, A. Ng, and G. Chiu, Phys. Rev. Lett. 78, 483 (1997).

${ }^{78}$ S. T. Weir, A. C. Mitchell, and W. J. Nellis, Phys. Rev. Lett. 76, 1860 (1996).

${ }^{79}$ N. C. Holmes, M. Ross, and W. J. Nellis, Phys. Rev. B 52, 15835 (1995).

${ }^{80}$ Tristan Guillot, Science 286, 72 (1999).

${ }^{81}$ W. J. Nellis, M. Ross, and N. C. Holmes, Science 269, 1249 (1995); W. J. Nellis, Chem.-Eur. J. 3, 192 (1997).

${ }^{82}$ M. Koenig, A. Benuzzi, B. Faral et al., "EOS Data Experiments for Plastic Foams Using Smoothed Laser Beams," Astrophy. J. Suppl. (to be published); Phys. Rev. Lett. 74, 2260 (1995).

${ }^{83}$ A. Benuzzi, T. Lower, M. Koenig, B. Faral, D. Batani, D. Beretta, C. Danson, and D. Pepler, Phys. Rev. E 54, 2162 (1996).

${ }^{84}$ A. M. Evans, N. J. Freeman, P. Graham, C. J. Horsfield, S. D. Rothman, B. R. Thomas, and A. J. Tyrrell, Laser Part. Beams 14, 113 (1996); S. D. Rothman and A. M. Evans, in CP429, Shock Compression of Condensed Matter-1997, edited by Schmidt, Dandekar, and J. Forbes (AIP, 1998), p. 79.

${ }^{85}$ T. Lower, V. N. Kondrashov, M. Basko, A. Kendl, J. Meyer-ter-Vehn, R. Sigel, and A. Ng, Phys. Rev. Lett. 80, 4000 (1998); M. Basko, Th. Lower,
V. N. Kondrashov, A. Kendl, R. Sigel, J. Meyer-ter-Vehn, Phys. Rev. E 56, 1019 (1997); T. A. Hall, A. Benuzzi, D. Batani, D. Beretta, S. Bossi, B. Faral, M. Koenig, J. Krishnan, Th. Lower, and M. Mahdieh, Phys. Rev. E 55, R6356 (1997).

${ }^{86}$ T. Lower, R. Sigel, K. Eidmann, I. B. Foldes, S. Huller, J. Massen, G. D. Tsakiris, S. Witkowski, W. Preuss, H. Nishimura, H. Shiraga, Y. Kato, S. Nakai, and T. Endo, Phys. Rev. Lett. 72, 3186 (1994).

${ }^{87}$ S. Fu, Y. Gu, J. Wu, and S. Wang, Phys. Plasmas 2, 3461 (1995).

${ }^{88}$ G. Fontaine and H. M. Van Horn, Astrophys. J., Suppl. 31, 467 (1976); G. Fontaine, H. C. Graboske, Jr., and H. M. Van Horn, Astrophys. J., Suppl. 35, 293 (1977).

${ }^{89}$ D. Saumon, private communication (2000).

${ }^{90}$ J. A. Paisner, E. M. Campbell, and W. J. Hogan, Fusion Technol. 26, 755 (1994).

${ }^{91}$ M. Andre, CHOCS No. 13, April 1995, Revue Scientifique et Technique de la Direction des Applications Militaires, CEA; M. Andre, "Conceptual Design of the French LMJ Laser,' First Annual International Conference on Solid State Lasers for Application to ICF, Monterey, CA, May 31June 1 (1995), SPIE proceeedings. 